Results Our primary study hypothesis is that diagnostic performance of VUBG is capable to differentiate fibroma and sarcoma with a sensitivity greater than 90\%. Considering as acceptable a sensitivity of $90 \%(\mathrm{H} 0)$ and excellent a sensitivity of $95 \%(\mathrm{H} 1)$, a sample size of 250 patients would be necessary to achieve $80 \%$ statistical power with a $5 \%$ type- 1 statistical error. Taking into account a potential drop-out rate of $10 \%$, there will needed 275 subjects to be included in our study. Primary study endpoint is sensitivity of VUGB anatomopathological diagnosis. Secondary endpoints include specificity, accuracy Youden's index, positive and negative predictive values.

Conclusion In case VUGB is demonstrated to be effective and safe to make the differential diagnosis, this should permit preoperatively the stratification of patients to either laparotomy for sarcomas or minimally invasive surgery for benign myomas. Therefore, both unnecessary laparotomies and cancerspoilage by sarcoma morcellations could be avoided at the maximum degree.

Disclosures Authors have nothing to disclose

\section{ANALYSIS OF UTERINE LAVAGE FOR EARLY OVARIAN CANCER DETECTION}

${ }^{1}$ Diana Zilovic, ${ }^{2}$ leva Vaicekauskaitè, ${ }^{3}$ Ruta Ciurliene, ${ }^{2}$ Rasa Sabaliauskaite, ${ }^{4}$ Sonata Jarmalaite. ${ }^{1}$ National Cancer Institute; Oncogyneacology, Vilnius, Lithuania; ${ }^{2}$ National Cancer Institute, Vilnius, Lithuania; Institute of Biosciences, Vilnius University, Vilnius, Lithuania; ${ }^{3}$ National cancer institute; National Cancer Institute, Vilnius, Lithuania; Oncogynecology, Vilnius, Lithuania; ${ }^{4}$ National Cancer Institute, Vilnius, Lithuania

\subsection{6/ijgc-2020-ESGO.200}

Introduction/Background Ovarian cancer (OC) has the highest mortality rate of all gynecologic. Currently there is no effective screening methodology or accurate early diagnostic test for OC. In recent years, it has been demonstrated that uterine lavage fluid could be useful for OC diagnosis and molecular profiling.

Methodology The aim of this study was to screen uterine lavage and ovarian tissue samples form Lithuanian OC patients for cancer-related mutations by targeted next generation sequencing (NGS) and to determine their associations with clinical features. DNA from 35 uterine lavage fluid from ovarian cancer, uterine cancer and benign ovarian mass patients and 20 ovarian tissue samples were analysed using NGS. The sequencing libraries were prepared using Ion AmpliSeq ${ }^{\mathrm{TM}}$ OnDemand Panel targeting 10 OC related genes: BRCA1, BRCA2, PIK3CA, PTEN, KRAS, TP53, CTNNB1, PPP2R1A, ARID1A and FBXW7. Variant uncertain significance (VUS) pathogenity predicted with VarSome database.

Results Technique of lavage from uterine cavity was successfully performed in all patients. We were able to detect 37 SNP (22 of these known to be pathogenic) in 20/35 uterine lavage samples, of these 19 (10 known pathogenic mutations) matched SNP found in tissue samples. 4/15 VUS predicted to be pathogenic: ARID1A c.5548delG, c.6628C > T, c.3606delG and BRCA1 c.3871delT. We were able to detect $62.5 \%(10 /$ 16) known pathogenic mutations in both matched samples ( $\mathrm{n}$ $=17$ ). Most mutations found in patients with serous OC and metastases.

Conclusion Cell-free DNA samples obtained from uterine lavage could be used for molecular profiling of OC patients. Uterine lavage is a simple procedure which can be performed in a physician's office-based setting and it holds great potential and significant promise for earlier diagnosis of OC and suggest the future possibility of this approach for screening women for gynecological cancers.

Disclosures This study is supported by NCI research fund.

\section{Late breaking abstracts}

\section{Breast cancer}

\section{EFFECT OF SENTINEL LYMPH NODE BIOPSY ON UPPER LIMB FUNCTION IN WOMEN WITH EARLY BREAST CANCER: A SYSTEMATIC REVIEW OF CLINICAL TRIALS}

Taynara Louisi Pilger, Daniely Franco Francisco, Francisco Jose Candido Dos Reis. Ribeirão Preto Medical School/University of São Paulo, Ribeirão Preto

\subsection{6/ijgc-2020-ESGO.201}

Introduction/Background Axillary surgery is essential in the management of early breast cancer. Conservative procedures like sentinel lymph node biopsy (SLNB) are less invasive than the traditional axillary dissection. However, some extent of ipsilateral upper limb dysfunction might still occur. The aim of this systematic review was to describe the incidence of lymphedema, pain, sensory, and motor disorders after SLNB in women with breast cancer.

Methodology We conducted a systematic review of randomized controlled trials. The search was performed on Pubmed, EMBASE, CINAHAL, and Web of Science. The search was based on the following concepts: breast cancer, sentinel lymph node biopsy, axillary dissection, upper limb complications. The risk of bias was evaluated using the Cochrane Rob 2.0 toll.

Conclusion SLNB is associated with postoperative complications that persist up to at least two years after the surgical procedure. The burden of complications, although lower when compared to axillary dissection, should not be ignored.

Disclosures The authors have no conflict of interest to disclose.

\section{Cervical cancer}

\section{CLINICAL CHARACTERISTICS, TREATMENT RESPONSE AND PROGNOSIS OF LOCALLY ADVANCED ADENOCARCINOMA OF THE CERVIX, A LOCAL STUDY}

Marilou Yu, Jonalyn Bagadiong. Jose R. Reyes Memorial Medical Center; Dr. Jose Fabella Memorial Hospital; Obstetrics and Gynecology Section of Gynecologic Oncology and Trophoblastic Diseases

\subsection{6/ijgc-2020-ESGO.202}

Introduction/Background Treatment of locally advanced cervical carcinoma regardless of histology, either, squamous, adenoor adenosquamous carcinoma is the same, concurrent chemoradiotherapy. Nevertheless, studies have different and contradictory results regarding the impact of tumor histology in relation to treatment response. The current study sought to determine the clinical characteristics, treatment response and prognosis of locally advanced adenocarcinoma of the cervix in comparison to squamous cell carcinoma.

Methodology Records of the cervical cancer patients from the outpatient department of the Section of Gynecologic Oncology of a tertiary hospital were retrospectively reviewed. 